\title{
Developing and implementing guidelines on culturally adapting the Addenbrooke's cognitive examination version III (ACE-III): a qualitative illustration
}

Waquas Waheed ${ }^{1}$, Nadine Mirza ${ }^{1 *}$ (D), Muhammed Wali Waheed ${ }^{2}$, Abid Malik $^{3}$ and Maria Panagioti ${ }^{1}$

\begin{abstract}
Background: Cognitive tests currently used in healthcare and research settings do not account for bias in performance that arises due to cultural context. At present there are no universally accepted steps or minimum criteria for culturally adapting cognitive tests. We propose a methodology for developing specific guidelines to culturally adapt a specific cognitive test and used this to develop guidelines for the ACE-III. We then demonstrated their implementation by using them to produce an ACE-III Urdu for a British South Asian population.

Methods: This was a several stage qualitative study. We combined information from our systematic review on the translation and cultural adaptation of the ACE-III with feedback from previous ACE-III adaptors. This identified steps for cultural adaptation. We formatted these into question-by-question guidelines. These guidelines, along with feedback from focus groups with potential users were used to develop ACE-III Urdu questions. Clinical experts reviewed these questions to finalise an ACE-III Urdu.

Results: Our systematic review found 32 adaptations and we received feedback from seven adaptors to develop the guidelines. With these guidelines and two focus groups with 12 participants a sample ACE-III Urdu was developed. A consensus meeting of two psychiatrists with a South Asian background and familiarity with cognitive tests and cultural adaptation finalised the ACE-III Urdu.

Conclusions: We developed a set of guidelines for culturally adapting the ACE-III that can be used by future adaptors for their own language or cultural context. We demonstrated how guidelines on cultural adaptation can be developed for any cognitive test and how they can be used to adapt it.
\end{abstract}

Keywords: Cross cultural, Ethnic minority, Non-English, Language, Psychometrics, Scale development, Transcultural, Translation

\footnotetext{
* Correspondence: nadine.mirza@postgrad.manchester.ac.uk

1 Centre for Primary Care and Health Services Research, The University of

Manchester, Suite 8, 5th Floor, Williamson Building Oxford Road, Manchester M13 9PL, UK

Full list of author information is available at the end of the article
}

(C) The Author(s). 2020 Open Access This article is licensed under a Creative Commons Attribution 4.0 International License, which permits use, sharing, adaptation, distribution and reproduction in any medium or format, as long as you give appropriate credit to the original author(s) and the source, provide a link to the Creative Commons licence, and indicate if changes were made. The images or other third party material in this article are included in the article's Creative Commons licence, unless indicated otherwise in a credit line to the material. If material is not included in the article's Creative Commons licence and your intended use is not permitted by statutory regulation or exceeds the permitted use, you will need to obtain permission directly from the copyright holder. To view a copy of this licence, visit http://creativecommons.org/licenses/by/4.0/. The Creative Commons Public Domain Dedication waiver (http://creativecommons.org/publicdomain/zero/1.0/) applies to the data made available in this article, unless otherwise stated in a credit line to the data. 


\section{Background}

There is an increasing prevalence of dementia on a global scale, with numbers showing there will be a rise from 50 million to 82 million in the next decade [1]. Within the United Kingdom (UK) there was an estimated 850, 000 people with dementia in 2015 but this is expected to increase to 1 million by 2021 and over 2 million by 2051 [2]. Thus, the need for early and accurate diagnosis continues to take precedence $[3,4]$.

In addition to a physical examination and robust interviewing [5] the diagnosis must be supplemented with the administration of a cognitive test [6]. These cognitive tests are characterised by their ability to either screen for or diagnose cognitive impairment and dementia [3]. In many cases they will also investigate severity and the potential subtype of dementia [7].

This is achieved through questions that assess individual cognitive capabilities- otherwise known as cognitive domains- such as attention and orientation, fluency, memory, language, and visuospatial abilities [5, 7]. They are often administered verbally, consisting of written, verbal, and sometimes physical tasks, ideally taking under $30 \mathrm{~min}$ [6].

Currently there are over 40 cognitive tests available and under use in both healthcare settings and within the context of research [8]. These range from brief screening tests such as the Six Item Cognitive Impairment Test (6 CIT) [9], Clock Drawing [10], the Mini Cog Test [11], and Test Your Memory (TYM) [12] to longer more extensive diagnostic tests such as the Mini Mental State Examination (MMSE) [13], the Montreal Cognitive Assessment (MoCA) [14], and the Addenbrooke's Cognitive Examination Version III (ACE-III) [15].

These cognitive tests were originally designed for English speaking European countries [16], standardised on well educated, male, Caucasian outpatients [17]. While applicable to that demographic, the original versions of these cognitive tests are not suitable for use with diverse populations [18].

Within the UK alone, 88 main languages other than English are spoken [19] and 8\% of the population do not have English as a first language [20]. It is also estimated that over 864,000 struggle to or are unable to speak English [20]. Cognitive tests in their current state would not be able to accommodate for these non-English speakers. Thus, many cognitive tests have been translated into targeted languages. However, these translated versions haven't necessarily been psychometrically validated and translation alone does not address gaps in understanding and acceptability that arise due to cultural context [4].

In the UK $14 \%$ of the population identifies as belonging to an ethnic minority group [19] and this is predicted to rise to $20 \%$ by 2051 [21]. A significant proportion of these ethnic minorities migrated to the
UK as young adults [22, 23]. Therefore, they still adhere in many ways to the culture of their home countries and pass this culture on to their children [22, 23].

This cultural context cannot be ignored when administering cognitive tests as culture influences how a respondent perceives test questions and how they respond to them, if they are even able to do so $[18,24]$. This is due to many cognitive test questions being reliant on a familiarity with the western calendar and western names, and knowledge of western history, objects and even wildlife [4]. When these questions have not been adapted to account for cultural context bias occurs. This equates to a loss of content equivalence (the questions are not relevant to the cultural context), criterion equivalence (the questions are unable to accurately assess for dementia) and content equivalence (the questions are no longer able to accurately assess the individual cognitive domains they were designed to) [25].

This accounts for higher rates of false positive and false negative scores across cognitive tests within non English speakers and ethnic minority groups as compared to their English speaking and Caucasian counterparts [18, 26, 27]. It also compromises the generalisability of the results of dementia research that incorporate these cognitive tests $[18,26,27]$.

To counter this, attempts at designing new cognitive tests for specific groups have been tried but this was deemed too time consuming and complex, reducing feasibility [28]. Another suggestion has been to adjust cut-off scores for different ethnic minority and nonEnglish speaking groups but this has been criticised for reducing sensitivity, specificity and likelihood ratios [29]. Therefore, culturally adapting an existing cognitive test has been regarded as a preferred alternative.

As mentioned, translating these tests does occur but this does not overcome the influence of culture beyond fluency in the target language. Existing cultural adaptations of cognitive tests do exist and have been developed through a variety of qualitative methods such as the use of global guidelines, involving experts and potential users in coproduction, and pilot testing [30]. However, at present there is no universal standard procedure or minimum criteria for culturally adapting these cognitive tests [31].

Therefore, there must be a global consensus on the steps and procedures that are essential for undertaking thorough cultural adaptation of cognitive tests [18]. These must be conducted before the adapted cognitive tests can be administered in healthcare and research settings. The final step would be a psychometric validation of the adapted cognitive test within the target population. However, prior to this there must be steps that focus on the cultural adaptation process itself.

We propose that for each cognitive test there should be a set of guidelines on how to culturally adapt the questions of that specific test. These would be developed through an 
incorporation of a review of previous literature on that test [25] with feedback from those who have already adapted it. These guidelines would provide step by step instructions on how to culturally adapt every question of that cognitive test in accordance with evidence to allow for the retention of content, criterion and conceptual equivalence [25].

The guidelines would then be implemented by using them to create culturally adapted versions of the cognitive test questions for a particular target demographic. These versions would be presented to potential users from the target demographic and clinical experts in the field. Their feedback would finalise which versions of the test questions will be retained in the adapted version of the cognitive test.
Following this there would be a cultural validation of the adapted cognitive test. This would consist of administering it to members of the target population and conducting qualitative cognitive interviews to assess the adapted cognitive tests understanding and acceptability within this population [32]. Once this has occurred, as mentioned, the psychometric validation would take place.

In this paper we will detail the process of developing and implementing such a set of guidelines for a nonEnglish speaking ethnic minority group within the UK. The cognitive test we selected was the ACE-III [15], a gold standard tool for the diagnostic accuracy of cognitive impairment and dementia [33], consisting of 19 questions that assess the cognitive domains attention,

Table 1 Questions of the Addenbrooke's Cognitive Examination Version III

\begin{tabular}{|c|c|}
\hline Question Number & Task/Question \\
\hline 1: Attention - Orientation & Ask the day, date, month, year, season, floor, street/hospital, town, county and country. \\
\hline 2: Attention - Registration & Say the words lemon, key and ball and ask them to repeat and try to remember. \\
\hline 3. Attention - Concentration & Ask to take 7 away from 100 and keep taking 7 away from the new number for 5 trials (Serial 7's). \\
\hline 4. Memory -Recall & Ask for the three words from 2. Attention - Registration. \\
\hline 5a. Fluency -Letters & $\begin{array}{l}\text { Ask for as many words as they can think of starting with the letter ' } \mathrm{P} \text { ', not including names of pronouns, in } \\
\text { one minute. }\end{array}$ \\
\hline 5b. Fluency -Animals & Ask for the names of as many animals as they can think of in one minute. \\
\hline 6. Memory -Anterograde & $\begin{array}{l}\text { Say the name and address 'Harry Barnes, } 73 \text {, Orchard Close, Kingsbridge, Devon' and ask them to repeat and } \\
\text { try to remember. }\end{array}$ \\
\hline 7. Memory -Retrograde & $\begin{array}{l}\text { Ask for the name of the current Prime Minister, name of the woman who was Prime Minister, name of the } \\
\text { USA president and name of the USA president who was assassinated in the 1960s. }\end{array}$ \\
\hline 8. Language -Comprehension & $\begin{array}{l}\text { Place a pencil and paper in front. Ask to 'place the paper on top of the pencil', 'pick up the pencil but not } \\
\text { the paper' and 'pass me the pencil after touching the paper'. }\end{array}$ \\
\hline 9. Language -Writing & $\begin{array}{l}\text { Ask to write two or more complete sentences about their last holiday/weekend/Christmas, without using } \\
\text { abbreviations. }\end{array}$ \\
\hline 10. Language -Repetition & Say the words caterpillar, eccentricity, unintelligible and statistician and ask them to repeat. \\
\hline 11. Language -Repetition & Say the proverbs 'All that glitters is not gold' and 'A stitch in time saves nine' and ask them to repeat. \\
\hline 12. Language -Naming & Show 12 images and ask them to name each. \\
\hline 13. Language -Comprehension & $\begin{array}{l}\text { Ask to point to 'the one which is associated with the monarchy', 'the one which is a marsupial', 'the one } \\
\text { which is found in the Antarctic' and 'the one which has a nautical connection' from the } 12 \text { images provided. }\end{array}$ \\
\hline 14. Language -Reading & Ask them to read the words sew, pint, soot, dough and height. \\
\hline $\begin{array}{l}\text { 15a. Visuospatial Abilities - Infinity } \\
\text { Diagram }\end{array}$ & the follow \\
\hline \multicolumn{2}{|l|}{ 15b. Visuospatial Abilities - Wire Cube A } \\
\hline \multicolumn{2}{|l|}{ 15c. Visuospatial Abilities - Clock } \\
\hline 16. Visuospatial Abilities & Ask them to count the number of dots without pointing. \\
\hline 17. Visuospatial Abilities & Ask them to identify the fragmented letters $\mathrm{K}, \mathrm{M}, \mathrm{A}$ and $\mathrm{T}$. \\
\hline 18. Memory -Recall & Ask for the three words from 6. Memory - Anterograde. \\
\hline 19. Memory - Recognition & $\begin{array}{l}\text { For each word of the name and address that could not be recalled, give the options listed and ask to identify } \\
\text { which word it was. }\end{array}$ \\
\hline
\end{tabular}


memory, fluency, language and visuospatial abilities (See Table 1).

The ACE-III and its predecessors, the ACE [34] and ACE-Revised (ACE-R) [35] have been translated into a range of languages and incorporated into use across the globe. English versions of the ACE-III have also been adapted for the UK and the United States of America (USA). However, the ACE-III was originally designed for English speakers native to Australia, with a reliance on knowledge of the cultural background [15] and although cultural adaptation has been undertaken by adaptors to produce suitable adaptations [36-38] there are no existing standardised guidelines for the cultural adaptation of this cognitive test.

As South Asians are the UKs largest ethnic minority group, at over $6.3 \%$ of the overall population, we selected them as our target population to culturally adapt for. The language we chose to adapt in was Urdu [32], a popular South Asian language and the 4th most common language spoken in the UK [19]. Prior to this there was only one other Urdu version of the ACE-III available, which was culturally adapted for use within India [39]. Due to this it was not applicable to the cultural contexts of other South Asian countries where Urdu speakers reside [40], nor to the Urdu speaking diaspora within Canada, the Middle East, the US and the UK [41].

Though this paper only details the development and implementation process of the guidelines, the ACE-III Urdu we produced through the methods has also undergone the cultural validation process, described elsewhere
[32]. This process undertook 25 cognitive interviews with cognitively healthy Urdu speaking British South Asians over the age of 60 . The ACE-III Urdu will now need to undergo a psychometric validation before being made available for widespread use.

\section{Methods}

A several stage qualitative approach was undertaken to develop guidelines for translating and culturally adapting the ACE-III (See Fig. 1) and implement them to develop an ACE-III Urdu (See Fig. 2):

Step 1: A systematic review.

Step 2: Receiving feedback from previous ACE-III adaptors.

Step 3: Collating the data to form guidelines.

Step 4: Implementing guidelines with feedback from lay persons and clinical experts.

Step 5: Developing the ACE-III Urdu.

\section{Step 1: systematic review}

We conducted a systematic review of all existing primary publications of translations and cultural adaptations of the ACE-III as well as its predecessors [30].

The electronic databases EMBASE, Medline, and PsychINFO were searched using the search terms "addenbrooke's cognitive examination or ace-iii or screen" or test or instrument or measure or tool or diagnos", "dementia or Alzheimer" or cognitive"*" and "sensitivity and specificity or accuracy or cut-off or receiver operator or ROC or Youden" [30]. We searched from January 2013, the year the ACE-III was introduced [15], to December 2016, which was when this review was conducted.

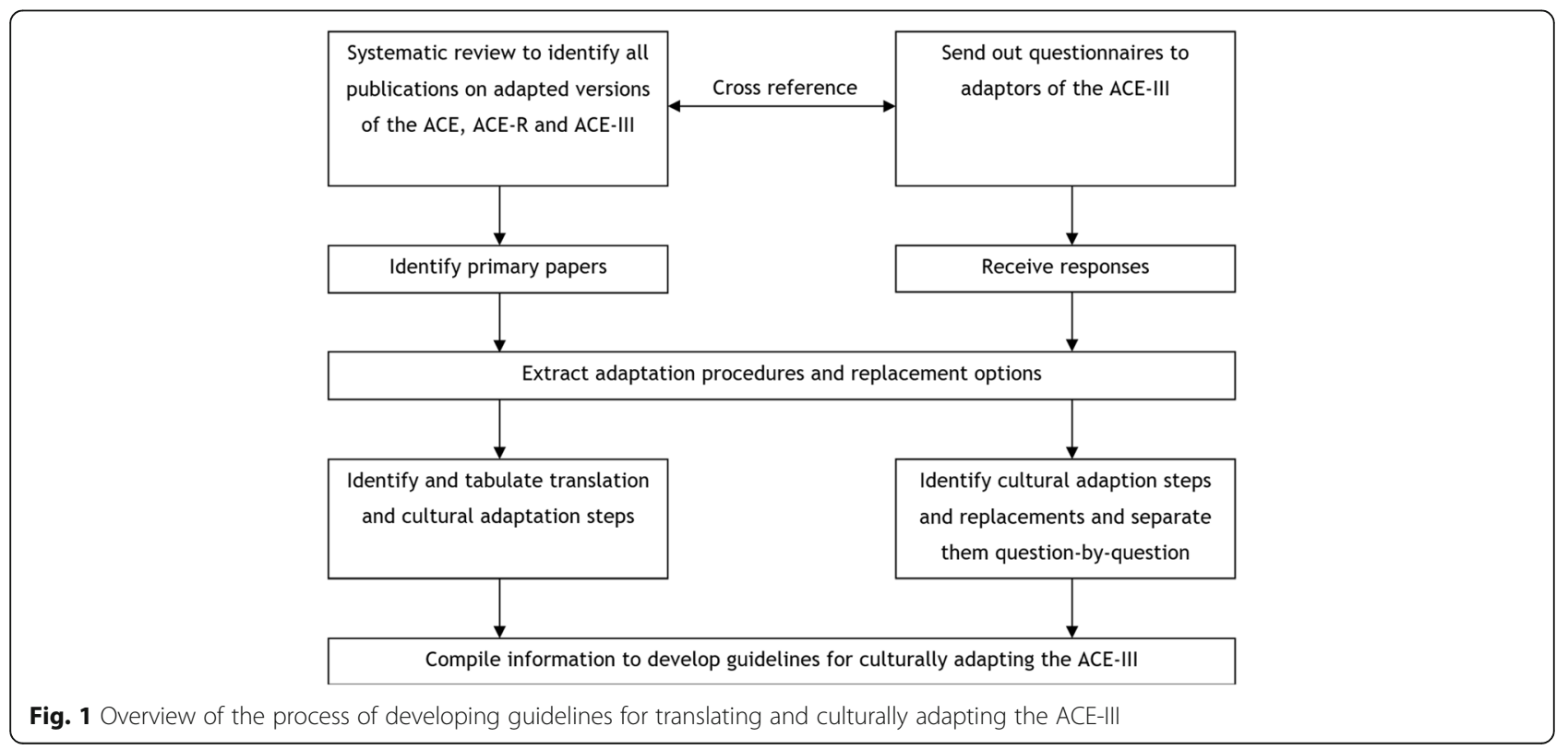


Develop potential questions for ACE-III Urdu to present to lay persons

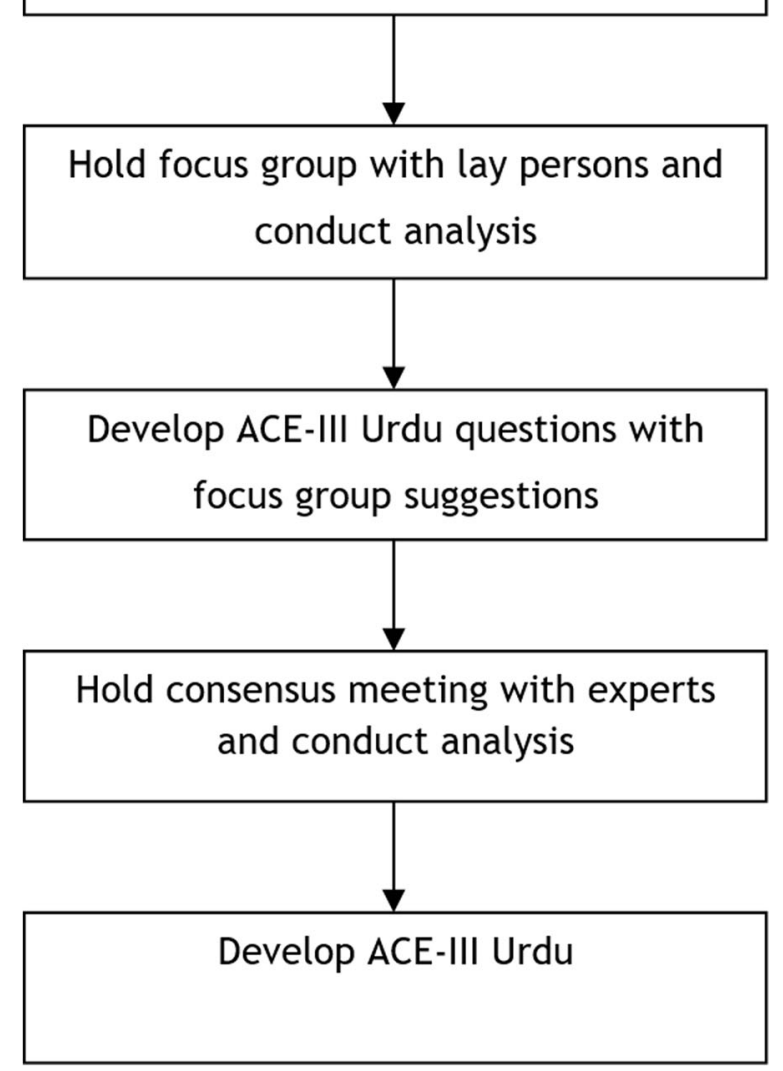

Fig. 2 Overview of the process of utilising the guidelines to culturally adapt the ACE-III for British South Asians

SCOPUS was also searched for any publication that cited the original paper [15]. Additionally, we also screened all included and excluded publications of a meta-analysis of the ACE and ACE-Revised (ACE-R) [42].

Publications, including validation studies, that incorporated the use of a translated or culturally adapted version of the ACE, ACE-R and ACE-III, from English into any other language, and were the primary source of that version, were included. For each of the publications we extracted data on the version of the ACE that was culturally adapted, the language it was translated into, the country it was culturally adapted for and the section of the text that described how it was culturally adapted. These reported processes were broken down into individual steps and grouped by which ACE-III question they described adapting. This allowed us to identify which questions were dependent on culture, how they had been culturally adapted, and what the rationale was behind the changes.
We also assessed the quality of the reported cultural adaptation of each publication with the Manchester Cultural Adaptation Reporting Questionnaire (MCAR) [30]. This shows which publications reported their cultural adaptation process in sufficient detail to be replicated by future adaptors.

\section{Step 2: feedback from adaptors of the ACE-III}

We aimed to receive feedback from official adaptors of the ACE-III; those who had translated and culturally adapted it for their respective language and culture and had made their adaptation available on the Neuroscience Research Australia (NeuRA) website that hosted the original ACE-III and its implementation materials at the time of this research [39].

We downloaded all available adaptations of the ACEIII from the website. We translated them into English through the use of an online translation application and, when available, with the aid of postgraduate research students at the University of Manchester who were native speakers of the languages. The translated adaptations were read through to identify which questions in each adaptation of the ACE-III had been culturally adapted beyond a translation verbatim. For each cultural adaptation we developed a questionnaire that highlighted the questions that had been culturally adapted along with the original ACE-III counterparts (See Additional file 1 for sample questionnaire). We asked for the rationale behind changing the original question and the development process of the culturally adapted version of the question with the rationale.

We distributed the questionnaires to the corresponding adaptors attached with a standardised email relaying the purpose of the questionnaires and a request for their completion. We also requested a check of our translation of their language version. After a two week period adaptors were sent a follow up email to act as a reminder. If adaptors did not initiate any form of contact after this no further contact was made.

\section{Step 3: data analysis and synthesis}

To develop guidelines for culturally adapting the ACEIII we collated the information from our systematic review [30] and the feedback from adaptors. We identified sets of mutually exclusive steps for culturally adapting each question of the ACE-III.

From our systematic review we had broken down the cultural adaptation processes extracted from each publication according to ACE-III question. The adaptation processes for each question across publications were merged and duplicates removed to identify the mutually exclusive steps that could be undertaken to adapt each question. 
The questionnaires sent to adaptors were already organised by question. We merged the adaptors' feedback on the cultural adaptation process of each question. We removed duplicating information so each question had mutually exclusive steps that could be undertaken to adapt that question along with the adaptors' accompanying rationale.

The cultural adaptation steps for each ACE-III question identified from the systematic review and from the adaptors' feedback were merged. Duplicates were removed to identify overall mutually exclusive cultural adaptation steps for each question. Accompanying rationale was presented with these steps and the respective publications and adapted versions of the ACE-III were cited, resulting in a question-by-question set of guidelines.

\section{Step 4: implementation of the guidelines}

We conducted two focus groups within the British South Asian community of Greater Manchester. We aimed to recruit 12-14 laymen participants overall, fluent in speaking and writing Urdu, over the age of 60, able to give informed consent and who did not have a history of cognitive impairment.

Participants were voluntarily recruited via convenience sampling from the local Pakistani Community Day Centre and provided with an information sheet, available in English and Urdu. They were given $24 \mathrm{~h}$ to decide if they wished to participate, after which they were contacted by a liaison at the Centre to confirm their participation and let them know the date and time of the focus group. These were also held at the centre. On the day of the focus group participants would be provided with consent forms and demographics sheets, available in English and Urdu.

Using the guidelines we produced several culturally adapted versions of all questions of the ACE-III, backed up by rationale, for the British Urdu speaking population. The most suitable option would need to be selected. We presented these versions of the questions within our focus groups to receive their feedback on the questions' cultural appropriateness, which versions should be retained for a potential ACE-III Urdu and whether they proposed any changes or suggestions of their own. This feedback was audio recorded and transcribed.

We also conducted a consensus meeting with clinical experts in the relevant fields, local to the Greater Manchester area. We aimed to recruit 2-4 experts on dementia, the cognitive testing process and the translation and cultural adaptation of these tests. They would also be familiar with the ACE-III, its rationale and how to administer it. These experts also had to be fluent in speaking and writing both English and Urdu and familiar with UK and South Asian cultures. The experts were recruited voluntarily via convenience sampling. The consensus meeting was held at the Centre for Primary Care and Health Services Research, at the University of Manchester, before which informed consent was obtained.

We presented the focus group feedback within the consensus meeting and experts determined which were the most culturally suitable adaptations of each question of the ACE-III. The consensus meeting was audio recorded, transcribed and the data was collated for each question of the ACE-III to determine how each would be culturally adapted.

\section{Step 5: developing the ACE-III Urdu}

To develop the ACE-III Urdu the template was acquired through NeuRA, allowing the ACE-III Urdu to retain the exact same format as the original ACE-III. Urdu is read from right to left so the template was reversed horizontally such that questions were presented on the right side of the template and the scoring instructions on the left side. Standard information requested prior to the administration of the ACE-III and instructions for the implementation of the questions were translated into Urdu and typed out. Each ACE-III question was typed out and designed according to the suggestions confirmed within the consensus meeting.

\section{Results}

\section{Step 1: systematic review}

We identified 113 publications through our search, of which 32 met out criteria for data extraction and analysis; 12 for the original ACE, 17 for the ACE Revised and 3 for the ACE-III. Overall, these publications spanned 18 languages; Arabic, Cantonese, Chinese, Czech, Danish, French, German, Greek, Hebrew, Italian, Japanese, Korean, Lithuanian, Malayalam, Persian, Portuguese, Slovak and Spanish [30].

The full results of the systematic review are described elsewhere [30].

\section{Step 2: feedback from adaptors of the ACE-III}

Our search of the NeuRA website identified 17 fully adapted versions of the ACE-III for the languages Egyptian Arabic, Saudi Arabian Arabic, Chinese, Estonian, Hebrew, Hindi, Hungarian, Indian Kannada, Italian, Japanese, Marathi, Polish, Portuguese, Spanish, Tamil, Telugu and Indian Urdu. Three versions had been retained in English but culturally adapted for India, New Zealand and the USA, resulting in a total of 20 ACE-III adaptations.

Of these adaptations the Estonian, Indian Kannada, Marathi, Japanese, Tamil and Telugu versions could not 
Table 2 Questions of the ACE-III that were culturally adapted by the adaptors

\begin{tabular}{|c|c|c|c|c|c|c|c|c|c|c|c|c|c|c|c|c|c|c|c|c|c|c|}
\hline \multicolumn{23}{|l|}{ ACE-III Questions } \\
\hline Language of the adaptors & 1 & 2 & 3 & 4 & $5 a$ & $5 b$ & 6 & 7 & 8 & 9 & 10 & 11 & 12 & 13 & 14 & $15 a$ & $15 b$ & $15 c$ & 16 & 17 & 18 & 19 \\
\hline Egyptian Arabic & & & & & $x$ & & $x$ & $x$ & & & $x$ & $x$ & $x$ & $x$ & $x$ & & & & & $x$ & $x$ & $x$ \\
\hline Hebrew & & $x$ & & & $x$ & & $x$ & $x$ & & & $x$ & $x$ & & & $x$ & & & & & & $x$ & $x$ \\
\hline Hindi & & & & & $x$ & & $x$ & $x$ & & & $x$ & $x$ & $x$ & $x$ & $x$ & & & & & $x$ & $x$ & $x$ \\
\hline Hungarian & & & & & & & $x$ & $x$ & & & $x$ & $x$ & & & $x$ & & & & & & $x$ & $x$ \\
\hline Polish & & $x$ & & & $x$ & & $x$ & $x$ & & & $x$ & $x$ & $x$ & $x$ & $x$ & & & & & & $x$ & $x$ \\
\hline Spanish & & $x$ & & & & & $x$ & $x$ & & & $x$ & $x$ & & $x$ & $x$ & & & & & & $\begin{array}{l}x \\
x\end{array}$ & $x$ \\
\hline Welsh & & $x$ & & & $x$ & & $x$ & $x$ & & & $x$ & $x$ & & & $x$ & & & & & & $x$ & $x$ \\
\hline
\end{tabular}

$\mathrm{x}=$ cultural adaptation was undertaken

be translated into English due to a lack of resources in terms of translation applications and translators. They excluded from our analysis (30\%). Questionnaires were developed for the remaining 14 adaptations (70\%) and distributed to their respective adaptors, of which a total of seven questionnaires (35\%) were returned to us fully completed.

The original Australian ACE-III was used by the Hindi, Hungarian and Spanish adaptors (15\%) and the UK version of the ACE-III was used by the Egyptian Arabic, Hebrew and Welsh adaptors (15\%) for their own adaptations. Polish adaptors used both (5\%).

Table 2 summarises which questions of the ACE-III were culturally adapted by which adaptors, thereby showing the frequency of reported cultural adaptation undertaken for each question. Table 3 shows examples of the culturally adapted versions of these questions across a range of languages and cultural contexts. We can see that all adaptors culturally adapted questions 6 , 7, 18 and 19 for memory and questions 10, 11 and 14 for language, and the majority had adapted question 2 for attention. In contrast, none of the adaptors had culturally adapted any of the questions assessing visuospatial abilities. This highlights which cognitive domains, and their respective questions, rely on culture and which would suffice with a simple translation into the target language.

\section{Step 3: data analysis and synthesis}

For each question of the ACE-III, the individual cultural adaptation steps identified from our systematic review and from adaptors' feedback, along with rationale undertaken, were tabulated to form the guidelines (See example in Fig. 3 and see Addtional file 2). For each question the following was presented:

Table 3 Examples of cultural adaptation of adapted ACE-III questions

\begin{tabular}{|c|c|c|}
\hline ACE-III Question & Example of adapted version & $\begin{array}{l}\text { Language } \\
\text { of version }\end{array}$ \\
\hline $\begin{array}{l}\text { 2: Attention - Registration: Say the words lemon, ask them to repeat and try to } \\
\text { remember. }\end{array}$ & Lemon was changed to Sliwka (Plum). & Polish \\
\hline $\begin{array}{l}\text { 5a. Fluency -Letters: Ask for as many words as they can think of starting with the } \\
\text { letter ' } P \text { ', not including names of pronouns, in one minute. }\end{array}$ & 'P' sound was changed to 'Sh' sound. & $\begin{array}{l}\text { Egyptian } \\
\text { Arabic }\end{array}$ \\
\hline $\begin{array}{l}\text { 6. Memory -Anterograde: Say the name and address 'Harry Barnes, } 73 \text {, Orchard Close, } \\
\text { Kingsbridge, Devon' and ask them to repeat and try to remember. }\end{array}$ & $\begin{array}{l}\text { The name and address were changed to } \\
\text { 'Katona Péter, Tavasz utca 42., Gyöngyös, } \\
\text { Heves megye'. }\end{array}$ & Hungarian \\
\hline 7. Memory -Retrograde: Ask for the name of the current Prime Minister. & Asked for the name of the current President. & Spanish \\
\hline 10. Language -Repetition: Say the word caterpillar. & $\begin{array}{l}\text { Caterpillar was changed to Colomennod } \\
\text { (Pigeons). }\end{array}$ & Welsh \\
\hline $\begin{array}{l}\text { 11. Language -Repetition: Say the proverb 'A stitch in time saves nine' and ask them } \\
\text { to repeat. }\end{array}$ & $\begin{array}{l}\text { The proverb was changed to 'The orchestra } \\
\text { played and the audience applauded' }\end{array}$ & Hebrew \\
\hline 12. Language -Naming: Show image of kangaroo and ask them to name each. & The image was changed to a goat. & Hindi \\
\hline $\begin{array}{l}\text { 13. Language -Comprehension: Ask to point to 'the one which is a marsupial', from } \\
\text { the } 12 \text { images provided. }\end{array}$ & $\begin{array}{l}\text { The question was changed to point to 'the } \\
\text { one which flies'. }\end{array}$ & $\begin{array}{l}\text { Egyptian } \\
\text { Arabic }\end{array}$ \\
\hline 14. Language -Reading: Ask them to read the word height. & Height was changed to Zamarzniety (Frozen). & Welsh \\
\hline 17. Visuospatial Abilities: Ask them to identify the fragmented English letters. & The letters were changed to Hindi alphabet. & Hindi \\
\hline
\end{tabular}




\section{QUESTION 1. ATTENTION}

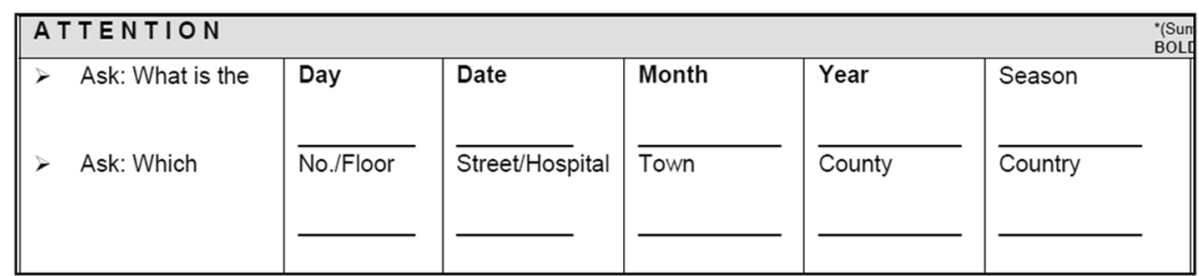

Often, the item is directly translated however some cultural adaptation may be necessary.

\begin{tabular}{|c|c|c|}
\hline $\begin{array}{l}\text { Culturally Adapted } \\
\text { Replacement }\end{array}$ & Language & Reasoning and Procedure \\
\hline 'County' became 'State' & Malayalam $^{1}$ & $\begin{array}{l}\text { Not all countries have counties so an } \\
\text { equivalent in the form of 'State' had } \\
\text { to be used. }\end{array}$ \\
\hline $\begin{array}{l}\text { Provided an example of } \\
\text { a country beforehand. }\end{array}$ & Malayalam $^{1}$ & $\begin{array}{l}\text { It was common to confuse state and } \\
\text { country so an example was given to } \\
\text { provide the concept of 'Country'. }\end{array}$ \\
\hline $\begin{array}{l}\text { The year, month and } \\
\text { date could tally with the } \\
\text { Lunar, Hindu or Muslim } \\
\text { calendar alternatively. }\end{array}$ & Malayalam $^{1}$ & $\begin{array}{l}\text { These are locally popular calendars } \\
\text { in some areas. }\end{array}$ \\
\hline $\begin{array}{l}\text { Vague description of } \\
\text { season or description of } \\
\text { weather as a response to } \\
\text { 'Season' was acceptable. }\end{array}$ & Malayalam $^{1}$, Czech $^{2}$ & $\begin{array}{l}\text { Some countries have seasons for } \\
\text { very brief periods of time so only } \\
\text { consider them in terms of weather. }\end{array}$ \\
\hline
\end{tabular}

Fig. 3 An example page of the guidelines

1) How the question has been previously culturally adapted with the steps undertaken.

2) Examples compiled from publications and the questionnaires, citing the respective languages and adaptors of the ACE-III

3) The rationale behind adapting the question and choosing the adapted replacement.

\section{Step 4: implementation of the guidelines}

Our focus groups had 12 voluntary participants, five female $(41 \%)$ and seven male (59\%), from ages $61-75$ years $(M=66.67, S D=6.44)$, from the Greater Manchester area. (See Table 4 for a breakdown of participant demographics). Seven participants were married, three were widowed, and two did not disclose. Six were retired, three were housewives, two were unemployed, and one did not disclose. Participants came from varied socioeconomic backgrounds, across a range of educational backgrounds.

Through our proposed suggestions for each question of the ACE-III Urdu, developed through the use of the guidelines, we determined questions $5 \mathrm{~b}, 8,9,15 \mathrm{a}, 15 \mathrm{~b}$,

Table 4 Demographic details of focus group participants

\begin{tabular}{lllll}
\hline PI & Age & Level of Education & First Language & $\begin{array}{l}\text { Other } \\
\text { Language }\end{array}$ \\
\hline IF & $60 \mathrm{~s}$ & $\mathrm{X}$ & Punjabi & Urdu, English \\
$2 \mathrm{~F}$ & $70 \mathrm{~s}$ & 10 th Year & Punjabi & Urdu \\
$3 \mathrm{~F}$ & $60 \mathrm{~s}$ & $\mathrm{FA}$ & Punjabi & Urdu \\
4F & $60 \mathrm{~s}$ & $\mathrm{FA}$ & Punjabi & Urdu \\
$5 \mathrm{~F}$ & $70 \mathrm{~s}$ & None & Punjabi & Urdu \\
$1 \mathrm{M}$ & $70 \mathrm{~s}$ & Graduate & Punjabi & Urdu, English \\
$2 \mathrm{M}$ & $60 \mathrm{~s}$ & BA & Urdu & English \\
$3 \mathrm{M}$ & $70 \mathrm{~s}$ & $\mathrm{MA}$ & Punjabi & Urdu, English, Arabic \\
$4 \mathrm{M}$ & $70 \mathrm{~s}$ & GCSEs & Urdu & English \\
$5 \mathrm{M}$ & $60 \mathrm{~s}$ & GCSEs & Urdu & Punjabi, English \\
$6 \mathrm{M}$ & $60 \mathrm{~s}$ & $\mathrm{X}$ & Punjabi & Urdu, Italian, English \\
& & Graduate & & \\
$7 \mathrm{M}$ & $70 \mathrm{~s}$ & Graduate & Punjabi & Urdu, English, Persian \\
\hline
\end{tabular}


Table 5 ACE-III Urdu items determined from focus groups and consensus meeting

\section{Suggestions \\ 1: Attention \\ i. Ask the question 'These days which of the four seasons is it?' ii. 'Hospital' and 'county' will be spelt using Urdu letters. \\ iii. Only accept dates in the English calendar. \\ 2: Attention \\ i. 'Lemon' is directly translated into Urdu. \\ ii. 'Key' is replaced with 'bell', spelt using Urdu letters. \\ iii. 'Ball' is directly translated into Urdu}

\section{3: Attention}

Use the word 'minus', spelt using Urdu letters and the Urdu translation for 'take away'.

\section{4: Memory}

Refer to Question 2: Attention

\section{5a: Fluency (Letters)}

Replace the letter ' $P$ ' with the Urdu letter ح゙ (chay).

\section{6: Memory}

i. The first name Haroon is used. The last name Butt is used. ii. The original ACE-III address will be retained and spelt using Urdu letters.

\section{7: Memory}

i. The first, second and third question of the original ACE-III, 'Name of the current Prime Minister', 'Name of the first female Prime Minister' and 'Name of the USA president' are retained.

ii. The fourth question will be replaced with 'Name of the princess who died in a car crash in the 1990s'.

\section{0: Language}

i. 'Eccentricity' is replaced with كُوش گَزار

ii. 'Unintelligible' is replaced with

$$
\text { نشى نب و فراز }
$$

iii. The Indian Urdu replacements for 'caterpillar' and 'statistician' are retained.

\section{1: Language}

The first saying, 'All that glitters is not gold', is translated into Urdu. The second saying is replaced with the saying that translated to 'You cannot clap with one hand'.

\section{2: Language}

i. Spoon is retained.

ii. Book is retained.

iii. Kangaroo is replaced with a goat.

iv. Penguin is replaced with a peacock.

$v$. Anchor is replaced with scissors.

\section{Justification}

Participants and experts agreed with the proposed suggestions developed with rationale from the guidelines.

Participants and experts agreed with the proposed suggestions developed with rationale from the guidelines for $\mathrm{i}$ and ii.

For iii, participants said 'bell' and 'ball' sound far too alike and it was decided that 'ball will be directly translated as the Urdu word for 'ball' is also one syllable.

Participants and experts agreed with the proposed suggestions developed with rationale from the guidelines.

Refer to Question 2: Attention

Participants agreed with both proposed suggestions developed with rationale from the guidelines.

Between the letters $ح$ (chay) and $\Sigma$ (gaaf), the former was then chosen by experts after debate due to its unique sound as the latter could be mistaken for the similar sounding letter $s$ (kaaf).

Participants agreed with all the proposed suggestions developed with rationale from the guidelines.

The name Haroon Butt was settled upon by the experts due to it retaining the sounds and length of the original name, Harry Barnes.

Participants agreed with the proposed suggestions developed with rationale from the guidelines.

The second question was retained according to the guidelines, despite what was said in the focus groups. Experts ruled that British Urdu speaking elderly should be aware of the first female Prime Minister of the UK due to her prominence. She would have been Prime Minister at the time when many of the elderly would have initially immigrated to the UK and would therefore know of her.

The fourth question was replaced with a new suggestion by the experts. Despite following the rationale of the guidelines 'Name of the British currency' and 'Name of the city where (a 'Wonder of the World') is located' were deemed too easy. It was also agreed that these replacements do not retain the conceptual equivalence of the question. 'Name of the princess who died in a car crash in the 1990' retains the concept, relating to a well know historical death relevant to the UK.

Participants agreed with the proposed suggestions developed with rationale from the guidelines.

'Eccentricity' was replaced with a new suggestion by experts as the words proposed were deemed too easy in comparison to the original counterparts.

Participants and experts agreed with the proposed suggestions developed with rationale from the guidelines.

Participants agreed with the proposed suggestions developed with rationale from the guidelines.

For iii, experts decided that though sheep are more common in the UK, British Urdu speakers would be familiar and able to recognise a goat. For iv, experts selected a peacock as British Urdu speakers would be 
Table 5 ACE-III Urdu items determined from focus groups and consensus meeting (Continued)

\begin{tabular}{l} 
Suggestions \\
\hline vi. Camel is retained. \\
vii. Harp is replaced with a dohl. \\
viii. Rhino is replaced with a bear. \\
ix. Barrel is replaced with a suitcase. \\
x. Crown is replaced with a cap. \\
xi. Crocodile is replaced with a tortoise. \\
xii. Accordion is replaced with a trumpet.
\end{tabular}

\section{3: Language}

The following questions were asked regarding the images: Which one is related to the head', 'Which one is found in the desert', 'Which one has a shell on it' and 'Which one is related to travel'.

\section{4: Language}

The words used in the Indian Urdu ACE-III were retained.

\section{7: Visuospatial Abilities}

The letters إو و o were selected.

\section{8: Memory}

Refer to Question 6: Memory.

\section{9: Memory}

Refer to Question 6: Memory.

The names Jamal Butt and Haroon Khan replaced the original names for recognition.

\section{Justification}

more familiar with it as opposed to a parrot. For $v$, experts settled on scissors as they are a common household object.

For vii, experts selected a dohl due to familiarity with it in the culture. For viii, experts proposed the new suggestion of a bear to replace the rhino as it is a better known wild animal but still unique in the UK. Experts ruled that a lion could be confused with other big cats such as a tiger and a monkey is not as relative to the cultural context of the UK. For ix, experts selected a suitcase as it is a form of container with a specific purpose.

For $\mathrm{x}$, experts proposed the new suggestion of a cap as it is a better known form of headwear.

For xi, experts proposed the new suggestion of a tortoise, because it is a better known wild animal that would be better recognised by the British Urdu speaking elderly.

For xii, a trumpet was selected out of the proposed instruments by participants from the focus groups as it was considered the most uniquely shaped and easily recognisable by British Urdu speaking elderly.

All questions were developed by the authors NM and WW according to the images that were finalised, following the guidelines.

Participants and experts agreed with the proposed questions.

Participants and experts agreed with the proposed suggestions developed with rationale from the guidelines

Participants and experts agreed with the proposed suggestions developed with rationale from the guidelines

Refer to Question 6: Memory.

Refer to Question 6: Memory.

Participants agreed with the proposed suggestions developed with rationale from the guidelines.

The alternative names chosen by the experts retained the length, familiarity and number of syllables. 15c and 16 would suffice with a direct translation. The remaining questions required further cultural adaptation that were deliberated over during these focus groups (See Additional file 3 for the proposed suggestions, developed using the guidelines).

Our consensus meeting was attended by two experienced old age psychiatrists who were both bilingual British Pakistanis. They had lived in both the UK and Pakistan and were familiar with the cultures of both countries. They were also both involved in clinical and research work relevant to South Asian populations and were knowledgeable about cognitive assessments, the ACE-III and the translation and cultural adaptation of cognitive tests.

\section{Step 5: developing the ACE-III Urdu}

The suggestions finalised within this consensus meeting and incorporated to form the ACE-III Urdu [19] can be seen in Table 5.

\section{Discussion}

In this paper we detailed the methods involved in developing a set of guidelines to culturally adapt a specific cognitive test; in this instance, the ACE-III. Following this we also demonstrated how these guidelines can be implemented to develop a cultural adaptation of the test it was designed for, for a specific target population.

The combination of the systematic review and adaptors' feedback provided us with detailed information on the cultural adaptation of the ACE-III, which was used to develop question-by-question guidelines. These present culturally adapted versions of questions of the ACE-III, backed up by rationale.

We could potentially have incorporated more cultures and languages through translators but due to limited resources we were unable to produce questionnaires for 6 of the existing adaptations. We also had a poor return rate of questionnaires (35\%), reducing the amount of additional information we could have received and 
compiled into the guidelines. Since our review and this research more cultural adaptations of the ACE-III have also been released that could not be included at this time.

Therefore we must acknowledge that the usefulness of these guidelines, and any guidelines for cognitive tests developed via these methods, is dependent on how many language and cultural versions of that test have been developed and how accessible these versions are. They are also limited by how many current adaptors of these versions provide rich data on the rationale behind culturally adapting questions that is often not conveyed through publications alone.

However, the methods for forming these guidelines are designed to allow for necessary updates as more information on cultural adaptation is acquired. In this way newer additions can be made by simply adding in any additional cultural adaptation steps found from new publications on adaptations of the ACE-III. The inclusion of feedback from adaptors also ensures we are not limited strictly to just published information.

In addition, we were still able to account for 22 international languages and cultural contexts in our guidelines. Through the frequency of cultural adaptation across questions, evidenced in our systematic review [30] and from the feedback of the adaptors, we were also able to determine which questions would most likely require cultural adaptation and which could suffice with a simple translation. The guidelines highlight this. With the accompanying rationale these guidelines would allow future adaptors to conduct their own cultural adaptation of the ACE-III and we have demonstrated this through our cultural adaptation of the ACE-III for a British Urdu speaking population.

While utilising the guidelines to develop potential questions for an ACE-III Urdu [32] we acknowledged British South Asians' preferences for certain English words that are spelt with Urdu letters, such as, 'county', 'bell' and 'ball', as opposed to translating words into Urdu. This is attributed to the mixing of English and Urdu that occurs within British Urdu speaking communities. We also noted the influence of the structure in which sentences are presented in Urdu, and proposed the rephrasing of questions to avoid confusion. This can be seen with the elaboration of 'What is the season?' to 'Which of the four seasons is it?' due to the Urdu word for weather and season being the same.

We presented these suggestions within our focus groups, allowing us to gather feedback from men and women of a vast array of educational backgrounds within the British Urdu speaking community. Throughout the discussion participants were able to follow the rationale provided by the guidelines when proposing suggestions for the ACE-III Urdu. There was a notable insistence on cultural adaptation for questions assessing memory and language, with little focus on fluency and visuospatial abilities. This is not to say that questions of fluency and visuospatial abilities may not require cultural adaptation, only that for this target population they did not.

Following this we conducted a consensus meeting with experts to review suggestions for the ACE-III Urdu proposed during the focus groups. There was a general consensus with the suggestions proposed, barring a few items. Within 'Question 2: Attention' experts preferred 'ball' be translated directly into Urdu and in the case of 'Question 7: Memory - Retrograde' experts decided to retain three questions as they were. The proposed suggestions were deemed too easy and the original questions were at a specific level of difficulty that was required to measure retrograde memory.

Overall this demonstrated the usability of the guidelines and their role in developing cultural adaptations of cognitive test questions alongside both potential user and clinical expert feedback. These methods also allowed us to develop a familiarity for the cognitive test we were working with, and the intricacies of the various test questions, in a way that general guidelines do not allow.

Through our methods we also developed the first version of the ACE-III Urdu that can be used in the cultural context of the UK. As mentioned earlier the next step was to conduct a cultural validation, which assessed the ACE-III Urdu's understanding and acceptability across older Urdu speaking British South Asians [32]. Further efforts will now be undertaken to determine its performance in the detection of dementia through a psychometric validation.

Overall, these methods can be replicated for any cognitive test to develop guidelines for adaptation specific to that test. Similarly, they can also be implemented in the same manner for any target population.

\section{Conclusions}

The guidelines are the first of their kind, and we have provided an in depth account of the approach we undertook to develop them and implement them. This was not restricted to published literature but incorporated the first hand experiences of cultural adaptation by existing adaptors of the ACE-III. This accounted for adaptations that may not have had corresponding publications. Furthermore, instead of adhering to general guidelines on cultural adaptation, developing guidelines designed for the cultural adaptation of the ACE-III allowed for familiarity with its individual questions. 
These guidelines for the ACE-III can now be implemented in the same manner we conducted for other language and cultural groups, with the added incorporation of potential user and clinical expert feedback. The implications of this methodology can also be taken forward to develop guidelines in the same manner for other existing cognitive tests. This would help to alleviate issues with mismatched culture across diverse populations that we currently see within healthcare and research settings.

\section{Supplementary information}

Supplementary information accompanies this paper at https://doi.org/10. 1186/s12888-020-02893-6.

Additional file 1: Supplementary Material- Appendix A1. Sample

Questionnaire- "Cultural adaptation process of the Italian ACE-III".

Additional file 2: Supplementary Material- Appendix A2. The first edition of our guidelines on translating and culturally adapting the ACEIII.

Additional file 3: Supplementary Material- Appendix A3. Table on 'Proposed ACE-III questions developed from the guidelines'.

\section{Abbreviations}

UK: United Kingdom; 6 CIT: six item cognitive impairment test; TYM: Test Your Memory; MMSE: Mini Mental State Examination; MoCA: Montreal Cognitive Aessment; ACE-III: Addenbrooke's Cognitive Examination Version III; USA: United States of America; ACE-R: ACE Revised; MCAR: Manchester Cultural Adaptation Reporting Questionnaire; NeuRA: Neuroscience Research Australia

\section{Acknowledgements}

Alex J Mitchell, Andrew J Larner, Emilia Sitek, Gwerfyl Roberts, JP Newman, Jordia MA Antem, Norbert Kovacs, Shailaja Mekala, Shuvarna Alladi and Tarik Qassem in providing completed questionnaires.

Ms. Nadia Siddiqui, who acted as our liase for recruitment, Women's Voices and the Greater Manchester Pakistani Association (GMPA).

\section{Authors' contributions}

The research question was formulated and the study designed by WW and NM. The study was carried out by NM, with assistance from AM and MP, supervised by WW. The qualitative data analysis was conducted by NM, with assistance from MWW, supervised by WW. NM wrote the manuscript. All authors read ad approved the manuscript.

\section{Funding}

There were no sponsorship or funding arrangements relating to this research.

\section{Availability of data and materials Not Applicable.}

\section{Ethics approval and consent to participate}

Ethical approval was waived for this research by the University of Manchester Research Ethics Committee (UREC). This was because the portion of the research requiring participation of members of the public was an act of public involvement for research on developing an Urdu version of the ACE-III that was used in a cultural validation study [19]. However, informed consent was still obtained from all participants through a signed consent form.

\section{Consent for publication}

Not Applicable.

\section{Competing interests}

The authors declare that they have no competing interests.

\section{Author details}

${ }^{1}$ Centre for Primary Care and Health Services Research, The University of Manchester, Suite 8, 5th Floor, Williamson Building Oxford Road, Manchester M13 9PL, UK. ${ }^{2}$ The University of Leicester, Leicester, UK. ${ }^{3}$ Greater Manchester Mental Health NHS Foundation Trust, Salford, UK

Received: 13 August 2019 Accepted: 24 September 2020

Published online: 06 October 2020

\section{References}

1. World Health Organization. Dementia Key Facts. 2020.

2. Alzheimer's Society. Alzheimer's Society's view on demography. 2020.

3. Gracey DJ, Morris RG. Neuropsychological assessment in dementia. Psychiatry. 2007;6(12):498-502.

4. Parker C, Philp I. Screening for cognitive impairment among older people in black and minority ethnic groups. Age Ageing. 2004;33(5):447-52.

5. Kipps CM, Hodges JR. Cognitive assessment for clinicians. J Neurol Neurosurg Psychiatry. 2005;11(2):22-30.

6. Sheehan B. Assessment scales in dementia. Ther Adv Neurol Disord. 2012 Nov; $5(6): 349-58$

7. Prince $M$, Knapp M, Guerchet M, McCrone P, Prina M, Comas-Herrera A, Wittenberg R, Adelaja B, Hu B, King D, Rehill A, Salimkumar D. Dementia UK update. Alzheimer's Soc. 2014:1-79.

8. Tsoi KK, Chan JY, Hirai HW, Wong SY, Kwok TC. Cognitive tests to detect dementia: a systematic review and meta-analysis. JAMA Intern Med. 2015; 175(9):1450-8.

9. Brooke P, Bullock R. Validation of a 6 item cognitive impairment test with a view to primary care usage. Int J Geriatric Psychiatry. 1999:14(11):936-40.

10. Brodaty $\mathrm{H}$, Moore $\mathrm{CM}$. The clock drawing test for dementia of the Alzheimer's type: a comparison of three scoring methods in a memory disorders clinic. Int J Geriatric Psychiatry. 1997;12(6):619-27..

11. Borson S, Scanlan J, Brush M, Vitaliano P, Dokmak A. The mini-cog: a cognitive 'vital signs' measure for dementia screening in multi-lingual elderly. Int J Geriatric Psychiatry. 2000;15(11):1021-7.

12. Brown J, Pengas G, Dawson K, Brown LA, Clatworthy P. Self administered cognitive screening test (TYM) for detection of Alzheimer's disease: cross sectional study. Bmj. 2009;338:b2030.

13. Folstein MF, Folstein SE, McHugh PR. "Mini-mental state": a practical method for grading the cognitive state of patients for the clinician. J Psychiatr Res. 1975;12(3):189-98

14. Nasreddine ZS, Phillips NA, Bédirian V, Charbonneau S, Whitehead V, Collin I, Cummings $J$, Chertkow $\mathrm{H}$. The Montreal cognitive assessment, MoCA: a brief screening tool for mild cognitive impairment. J Am Geriatr Soc. 2005; 53(4):695-9.

15. Hsieh S, Schubert S, Hoon C, Mioshi E, Hodges JR. Validation of the Addenbrooke's cognitive examination III in frontotemporal dementia and Alzheimer's disease. Dement Geriatr Cogn Disord. 2013;36(3-4):242-50.

16. Tuerk R, Sauer J. Dementia in a black and minority ethnic population: characteristics of presentation to an inner London memory service. BJPsych bulletin. 2015 Aug;39(4):162-6.

17. Fillenbaum GA, Heyman A, Williams K, Prosnitz B, Burchett B. Sensitivity and specificity of standardized screens of cognitive impairment and dementia among elderly black and white community residents. J Clin Epidemiol. 1990 Jan 1:43(7):651-60

18. Ganguli M, Hendrie HC. Screening for cognitive impairment and depression in ethnically diverse older populations. Alzheimer Dis Assoc Disord. 2005; 19(4):275-8.

19. Office for National Statistics, National Records of Scotland, Northern Ireland Statistics and Research Agency. 2011 Census aggregate data. UK Data Service June 2016.

20. Mirza N. Those Leaflets Clinical Psychology Forum; 2019. p. 318

21. Rees $P$, Wohland $P$, Norman $P$, Boden $P$. A local analysis of ethnic group population trends and projections for the UK. J Popul Res. 2011;28(2-3):149-83.

22. Mukadam N, Cooper C, Livingston G. A systematic review of ethnicity and pathways to care in dementia. Int J Geriatric Psychiatry. 2011;26(1):12-20.

23. Rait G, Burns A, Baldwin R, Morley M, Chew-Graham C, St Leger AS. Validating screening instruments for cognitive impairment in older south Asians in the United Kingdom. Int J Geriatric Psychiatry. 2000;15(1):54-62.

24. Beaton DE, Bombardier C, Guillemin F, Ferraz MB. Guidelines for the process of cross-cultural adaptation of self-report measures. Spine. 2000;25(24):3186-91. 
25. Flaherty JA, Gaviria FM, Pathak D, Mitchell T, Wintrob R, Richman JA, Birz S Developing instruments for cross-cultural psychiatric research. J Nervous Mental Dis. 1988.

26. Khan F, Tadros G. Complexity in cognitive assessment of elderly British minority ethnic groups: cultural perspective. Dementia. 2014;13(4):467-82.

27. Richards MA, Abas ME. Cross-cultural approaches to dementia and depression. In Ethnicity: An Agenda for Mental Health. (D. Bhugra and V. Bahl, Eds). London: Gaskell.

28. Guillemin F, Bombardier C, Beaton D. Cross-cultural adaptation of healthrelated quality of life measures: literature review and proposed guidelines. J Clin Epidemiol. 1993:46(12):1417-32.

29. Bohnstedt M, Fox PJ, Kohatsu ND. Correlates of mini-mental status examination scores among elderly demented patients: the influence of race-ethnicity. J Clin Epidemiol. 1994;47(12):1381-7.

30. Mirza N, Panagioti M, Waheed MW, Waheed W. Reporting of the translation and cultural adaptation procedures of the Addenbrooke's cognitive examination version III (ACE-III) and its predecessors: a systematic review. BMC Med Res Methodol. 2017;17(1):141.

31. Maneesriwongul W, Dixon JK. Instrument translation process: a methods review. J Adv Nurs. 2004:48(2):175-86.

32. Mirza N, Panagioti M, Waheed W. Cultural validation of the Addenbrooke's cognitive examination version III Urdu for the British Urdu-speaking population: a qualitative assessment using cognitive interviewing. BMJ Open. 2018;8(12):e021057.

33. Cheung G, Clugston A, Croucher M, Malone D, Mau E, Sims A, Gee S. Performance of three cognitive screening tools in a sample of older new Zealanders. Int Psychogeriatr. 2015;27(6):981-9.

34. Mathuranath PS, Nestor PJ, Berrios GE, Rakowicz W, Hodges JR. A brief cognitive test battery to differentiate Alzheimer's disease and frontotemporal dementia. Neurology. 2000;55(11):1613-20.

35. Mioshi E, Dawson K, Mitchell J, Arnold R, Hodges JR. The Addenbrooke's cognitive examination revised (ACE-R): a brief cognitive test battery for dementia screening. Int J Geriatric Psychiatry. 2006:21(11):1078-85.

36. Machado A, Baeta É, Pimentel P, Peixoto B. Psychometric and normative indicators of the version of the Addenbrooke's cognitive examination-III. Preliminary study on a sample of health subjects Acta Neuropsychologica. 2015;13(2)

37. Guiu JM, de Bobadilla RF, Escudero G, Pérez JP, Cortés A, Rodríguez EM, Salgado MV, Ramos TM, Kulisevsky J, Guiu JM. Validación de la versión española del test Addenbrooke's Cognitive Examination III para el diagnóstico de demencia. Neurología: Publicación oficial de la Sociedad Española de Neurología. 2015;30(9):545-51.

38. Qassem T, Khater MS, Emara T, Tawfik HM, Rasheedy D, Mohammedin AS, Tolba MF, Aziz KA. Translation and cross cultural adaptation of the Addenbrooke's cognitive examination III into Egyptian Arabic In Royal College of Psychiatrists International Congress; 2014.

39. Neuroscience Research Australia. 2016. [online] Available at: https://www.neura edu.au/research-clinic/frontier/research/downloads/. [Accessed 10 Nov 2016].

40. Central Intelligence Agency Library. The World Factbook. 2016. [online] Available at: https://www.cia.gov/library/publications/the-world-factbook/ wfbExt/region_sas.html [Accessed 10 Nov 2016].

41. South Asian Concern. Who are South Asians. 2016. [online] Available at: http://southasianconcern.org/south-asians/who-are-south-asians/ [Accessed 10 Nov 2016]

42. Larner AJ, Mitchell AJ. A meta-analysis of the accuracy of the Addenbrooke's cognitive examination (ACE) and the Addenbrooke's cognitive examinationrevised (ACE-R) in the detection of dementia. Int Psychogeriatr. 2014 Apr; 26(4):555-63.

\section{Publisher's Note}

Springer Nature remains neutral with regard to jurisdictional claims in published maps and institutional affiliations.

Ready to submit your research? Choose BMC and benefit from:

- fast, convenient online submission

- thorough peer review by experienced researchers in your field

- rapid publication on acceptance

- support for research data, including large and complex data types

- gold Open Access which fosters wider collaboration and increased citations

- maximum visibility for your research: over $100 \mathrm{M}$ website views per year

At $\mathrm{BMC}$, research is always in progress.

Learn more biomedcentral.com/submissions 